\title{
Prácticas de gestión del conocimiento y trabajo en equipo en instituciones de educación superior: escalas de medición
}

\author{
Dayanna Sánchez-Rodríguez ${ }^{1}$, Julio C. Acosta-Prado ${ }^{2,3, *}$ y Arnold A. Tafur-Mendoza ${ }^{4}$ \\ (1) Dirección de Gestión de Investigación, Universidad Piloto de Colombia, 083060 Bogotá, Colombia. \\ (2) Facultad de Ciencias Empresariales, Universidad del Pacífico, 15072 Lima, Perú. \\ (3) Facultad de Administración de Empresas, Universidad Externado de Colombia, 111711 Bogotá, Colombia. \\ (4) Centro de Investigación, Universidad del Pacífico, 15072 Lima, Perú. \\ (correo-e: dsanchez@unipiloto.edu.co; jc.acostap@up.edu.pe; aa.tafurm@up.edu.pe)
}

Recibido Jun. 18, 2020; Aceptado Ago. 18, 2020; Versión final Sep. 1, 2020, Publicado Feb. 2021

\begin{abstract}
Resumen
El objetivo de este estudio fue desarrollar y analizar las propiedades psicométricas de dos escalas de medición que evalúen las prácticas de gestión del conocimiento y el trabajo en equipo. Se diseñó un estudio instrumental en una muestra no probabilística de 334 grupos de investigación en instituciones de educación superior colombianas. Se aplicaron las escalas de medición con 12 ítems para prácticas de gestión del conocimiento y de 18 ítems para trabajo en equipo. Los resultados confirman el buen ajuste de las prácticas de gestión del conocimiento a una estructura bifactorial y del trabajo en equipo a una estructura de tres factores. Se aportaron evidencias de validez basadas en el contenido y en la relación con otras variables. Asimismo, las puntuaciones obtuvieron adecuados niveles de fiabilidad. Se concluye que los puntajes de los grupos de investigación en las escalas desarrolladas poseen propiedades psicométricas adecuadas. Por tanto, estos instrumentos pueden ser utilizados en instituciones de educación superior.
\end{abstract}

\section{Knowledge management practices and teamwork in higher education institutions: measurement scales}

\begin{abstract}
The objective of this study was to develop and to analyze psychometric properties of two measurement scales that evaluate knowledge management practices and teamwork. An instrumental study was designed in a nonprobabilistic sample of 334 research groups at Colombian higher education institutions. Measurement scales with 12 items for knowledge management practices and 18 items for teamwork were applied. The results confirm satisfactory adjustment of knowledge management practices to a two-factor structure and of teamwork to a three-factor structure. Validity evidence was provided based on test content and on relationships to other variables. The scores showed adequate levels of reliability. It is concluded that research groups scores on the two scales developed here have adequate psychometric properties and that, therefore, higher education institutions can use these scales.
\end{abstract}




\section{INTRODUCCIÓN}

El rápido avance de las tecnologías de la información y comunicación ha llevado a que el ámbito educativo se transforme en lo que conocemos actualmente como sociedad del conocimiento, donde el conocimiento es el principal insumo para el desarrollo de una nación (Romo et al., 2012). Ante este panorama, las instituciones de educación superior (IES) y sus grupos de investigación tienen un rol fundamental en la generación y difusión del conocimiento en la sociedad, ya que la investigación es una actividad básica en el contexto universitario (Conchado et al., 2015). La investigación científica es una actividad orientada a la obtención de nuevos conocimientos para dar solución a problemas de carácter científico, buscando contribuir con aportes teórico-prácticos a la mejora de su actividad laboral, aumento de producción intelectual, solución puntual de problemas o la suma de estas. Por ello, los grupos de investigación son elementos clave, cuyos procesos de investigación deben ser analizados. Las características de sus actividades, están marcadas por la renovación constante, tanto de sus miembros como del entorno en el que se desenvuelven (Singh y Gupta, 2014).

Los grupos de investigación son los encargados de promover el conocimiento científico, estudiar diversos fenómenos, y, sobre todo, ser partícipes en la solución de las problemáticas de las empresas y de la sociedad. Los grupos de investigación son un conjunto de personas que interactúan entre sí con la finalidad de investigar y dar solución a una problemática explícita y definida en el campo donde se desempeñan, todo ello a partir de un plan de trabajo que les permite generar productos de conocimiento como artículos, normas, software, entre otros (Colciencias, 2018). En Colombia, según los datos de Colciencias en 2017 (Colciencias, 2018), de los 5207 grupos de investigación evaluados, la mayoría (41\%) se ubicó en la categoría C (2113 grupos). Esto refleja las deficiencias que presentan los grupos respecto al desarrollo de investigaciones. El modelo de medición de grupos de investigación de Colciencias busca conocer qué hacen, cómo lo hacen, qué tipos de productos generan, qué talento humano forman y cómo se relacionan entre sí. Sin embargo, este modelo no contempla la evaluación de las prácticas de gestión del conocimiento que los grupos realizan y el trabajo en equipo entre sus integrantes. Por ello, es necesario contar con instrumentos que midan estas variables para comprender mejor la dinámica de los grupos de investigación.

La gestión del conocimiento implica el manejo del conocimiento organizacional que mejora una variedad de características de los resultados obtenidos por una organización (Singh y Gupta, 2014). En este sentido, las organizaciones buscan aprovechar sus procesos y recursos internos, dando lugar a la gestión de la calidad total, los sistemas de expertos, la reingeniería de los procesos de negocio, las organizaciones de aprendizaje, el desarrollo de competencias básicas y el enfoque estratégico. Por otro lado, las prácticas de gestión del conocimiento constituyen todas las actividades que contribuyen al manejo intencionado de los recursos de conocimiento de una organización (Hussinki et al., 2017). Las prácticas de gestión del conocimiento ayudan a las IES a dinamizar y potenciar la producción científica en un contexto globalizado. De esta forma, se deben contar con instrumentos que evalúen estas prácticas, fundamentalmente en las etapas iniciales, donde se da la creación del conocimiento dentro de los grupos y sobre las que inciden las políticas que tienen las organizaciones a las que pertenecen (Lee y Wong, 2015). A partir de lo señalado, el presente estudio busca construir una escala que mida los aspectos indicados, por tanto, se han considerado dos dimensiones, la estrategia institucional y la creación de conocimiento.

Si bien existen estudios de la gestión del conocimiento enfocados en grupos de investigación (Argueta y Jiménez, 2017; Guevara, 2011), no se tienen en igual medida aquellos que se centren únicamente en las prácticas de gestión del conocimiento (García-Alisina y Gómez-Vargas, 2015). En el contexto de investigación dentro de las IES, se busca analizar la gestión del conocimiento científico, sumado al de tipo administrativo, relacionado a los procedimientos propios de los grupos de investigación (búsqueda de convocatorias, presentación de proyectos, diseño de proyectos, entre otras), que permiten la obtención de valor y ventajas competitivas (García-Alisina y Gómez-Vargas, 2015). Esto es lo que se conoce como estrategia institucional, ligada al planeamiento estratégico, implementación y actividades de actualización que consideren al conocimiento como el eje central. Uno de los problemas que presentan los grupos de investigación es el poco apoyo institucional que reciben por parte de las IES a las que pertenecen, referente a baja inversión en recursos tecnológicos, políticas excluyentes, bajo presupuesto asignado, falta de programas de capacitación y formación continua, inapropiados ambientes de trabajo e infraestructuras tecnológicas deficientes (Lin et al., 2014). Por ello, la estrategia institucional es considerada un componente de las prácticas de gestión del conocimiento que incide en el funcionamiento de los grupos de investigación.

Otro aspecto a considerar en los contextos de investigación son los estudios realizados sobre la gestión del conocimiento en IES, que se centra en analizar variables relacionadas a la creación de conocimiento (Casimir et al., 2012). La creación de conocimiento implica la generación del mismo a partir del conocimiento tácito o explícito, donde juega un rol importante tanto el trabajo intelectual como las acciones colectivas o de trabajo en conjunto (Ramayah et al., 2014). La creación del conocimiento es una actividad inherente a docentes e investigadores que laboran en IES dado que está implícita en su misión (Romo et al., 2012). Para la creación 
del conocimiento, se requiere una flexibilidad para manejar cualquier tipo de conocimiento. La creación del conocimiento científico no puede ser totalmente planificada y controlada, en muchas ocasiones, la innovación resulta imprevisible (Rodríguez et al., 2001). En este proceso de creación están inmersos aspectos susceptibles a considerar, tales como las formas de organización para la creación de conocimiento, tipos de productos de conocimiento, y el uso de herramientas y estrategias para generar conocimiento. De esta manera, la creación del conocimiento es un componente de las prácticas de gestión de conocimiento que se da en las etapas iniciales de todo proyecto de los grupos de investigación.

Por otro lado, los grupos de investigación están inmersos en procesos de socialización y desarrollo, creando las condiciones necesarias para desplegar su labor investigativa, teniendo como características dinámicas la integración social, la consolidación y la cohesión, que se reflejan en las relaciones entre los integrantes y el grado de integración entre ellos y en conjunto (Rey et al., 2008). Todo lo mencionado hace referencia al trabajo en equipo, considerada una variable fundamental para el logro de las actividades colectivas. El trabajo en equipo implica la sincronización eficaz de un grupo de personas que aportan mucho más que comportamientos directamente relacionados con la consecución de determinadas metas (Mendo-Lázaro et al., 2017). Los objetivos que persigue el trabajo en equipo pueden ser de naturaleza académica (habilidades intelectuales o resolución de problemas) o social (relaciones interpersonales, actitudes hacia los compañeros, entre otras). Dentro de los grupos de investigación, la formación académica de sus integrantes es relativamente homogénea, por el contrario, la dimensión social marca la diferencia entre un grupo y otro. Ante esto, se busca desarrollar una escala que mida el trabajo el equipo desde una perspectiva social, donde se analicen las competencias interpersonales y las habilidades sociales del equipo. De este modo, se han considerado tres dimensiones: comunidad de aprendizaje, colaboración y cooperación, y diversidad funcional.

Torres et al. (2014) señalan que es posible construir la base de una efectiva capacidad de organización a partir de la formación de comunidades de aprendizaje. A través de estas se logra codificar y comprender la dinámica de la interacción en los grupos de investigación. Propiciar espacios de encuentro permite la circulación de saberes, conversar sobre preguntas de investigación relevantes y los hallazgos obtenidos. De esta manera, en los grupos de investigación, estas comunidades de aprendizaje son lugares de encuentro entre pares, donde se demanda entrega y participación, escucha activa y toma de posturas, así como el diálogo que permite conocer las perspectivas de cada uno de los integrantes. Por tanto, las comunidades de aprendizaje, se transforman en mecanismos para la consolidación de estos espacios de diálogo (Gaviria et al., 2007). Por otra parte, dentro de los grupos de investigación, se dan a la vez procesos relacionados a cómo sus integrantes interactúan. La cooperación indica un proceso de trabajo conjunto, en tanto que, la colaboración tiene un significado mayor, donde la interacción del grupo se da a partir de objetivos comunes compartidos. Los grupos de investigación eficientes se caracterizan por tener mayores actividades colaborativas que cooperativas, donde los integrantes se ayudan entre sí para la propia creación, definición de roles y posterior crecimiento sobre las bases de colaboración, confianza y compatibilidad (Gómez y Acosta, 2003). La cooperación y colaboración son aspectos inherentes al trabajo en equipo.

Por último, la existencia de diferentes y diversos roles, perfiles, funciones y responsabilidades en los equipos de investigación, incorporando diferencias del capital intelectual contenido en la estructura, y en el entorno es lo que se conoce como diversidad funcional. Las organizaciones con orientación al fomento del conocimiento e innovación se caracterizan por conformar estructuras orientadas a sistemas multinivel, donde los integrantes construyen entornos de actividades y tareas que impulsan la dificultad, la complejidad y el ritmo de la tarea del equipo; razón por la que es necesario entender el contexto del sistema y los vínculos a través de múltiples niveles (persona-equipo-organización) como fuentes clave de contingencias o demandas que requieren de procesos de equipo alineados (Rey et al., 2008).

Diversos instrumentos se han diseñado para medir aspectos de la gestión del conocimiento en los últimos años. Entre ellos se encuentra una escala que mide las condiciones de la gestión del conocimiento en empresas con base tecnológica (Acosta-Prado et al., 2020) a través de cinco componentes, siendo uno de estos el propósito estratégico; la escala cuenta con evidencia de validez basada en la estructura interna y adecuados niveles de fiabilidad ( $\omega>$.80). Paez-Logreira et al. (2016) desarrollaron un instrumento para medir las percepciones de la gestión del conocimiento en grupos de investigación a través de nueve ítems; no obstante, esta encuesta no cuenta con estudios psicométricos. La única escala encontrada para la medición de las prácticas de gestión del conocimiento fue elaborada por Guevara (2011), quien construyó de seis ítems para evaluar este constructo. Sin embargo, para la obtención de evidencias de validez realizaron una evaluación piloto a miembros de grupos de investigación solo para mejorar la claridad de los ítems, mientras que, la confiabilidad no fue estimada por ningún método. Por tanto, el instrumento presentado por Guevara (2011) carece de un estudio formal para la evaluación de sus propiedades psicométricas.

En relación a los instrumentos de medición construidos para evaluar el trabajo en equipo, no se encontró alguno que haya sido validado en grupos de investigación. La mayor parte de estos han sido desarrollados 
en estudiantes universitarios para conocer sus actitudes, competencias, autoeficacia y percepciones sobre el trabajo en equipo. Mendo-Lázaro et al. (2017) diseñaron una escala para medir las actitudes hacia el trabajo en equipo a través de 12 ítems, contando con un buen ajuste a dos factores (actitud académica y socialafectiva), altos niveles de fiabilidad por consistencia interna $(\alpha>.80)$ y estabilidad temporal $(r=.870)$, e invarianza factorial según el género. Aguado et al. (2014) construyeron una prueba para evaluar las competencias dentro del trabajo en equipo, compuesta por 36 ítems agrupados en ocho dimensiones; la prueba cuenta con evidencia de validez basada en el contenido y estructura interna del test, así como un índice de fiabilidad satisfactorio en la escala total $(\alpha=.89)$. Gastélum et al. (2012) diseñaron una escala de 16 ítems para medir la autoeficacia en los trabajos en equipo, este instrumento presentó adecuadas propiedades psicométricas, a partir de la evidencia de validez basada en la estructura interna y fiabilidad por consistencia interna $(\alpha>.90)$. Además, Ramírez et al. (2012) construyeron un cuestionario para evaluar la percepción del trabajo en equipo mediante 27 ítems y compuesto teóricamente por cinco dimensiones, integración, organización, interacción, funcionamiento y calidad de los resultados; sin embargo, no se reportaron evidencias de su calidad psicométrica.

Con la finalidad de cubrir esta brecha de conocimiento, el objetivo principal del presente estudio fue desarrollar y analizar las propiedades psicométricas de dos escalas de medición que evalúen las prácticas de gestión del conocimiento y el trabajo en equipo en grupos de investigación de IES colombianas. Estas escalas buscan contribuir a la comprensión de estos dos constructos en el contexto de investigación en IES. Las prácticas de gestión del conocimiento se enfocaron en las primeras etapas donde se desarrolla el conocimiento, desde una mirada interna (creación de conocimiento) como externa (estrategia institucional). Por otro lado, el trabajo en equipo estuvo orientado al desarrollo de habilidades interpersonales y el logro de objetivos colectivos, siendo las dimensiones de comunidad de aprendizaje, colaboración y cooperación, y diversidad funcional, aquellas que mejor reflejan estos dos aspectos. La construcción de ambas escalas permitirá contar con instrumentos apropiados para la evaluación de grupos de investigación, principalmente en contextos de educación superior, que cada año van en aumento debido a las políticas en investigación de diversos países latinoamericanos. La evaluación de las prácticas de gestión del conocimiento y el trabajo en equipo en los grupos de investigación ayudará a describir su funcionamiento, estimar la influencia sobre sus resultados académicos y ayudar en la toma de decisiones a nivel institucional o gubernamental.

\section{MÉTODO}

El estudio fue de clase instrumental (Ato et al., 2013), donde las propiedades psicométricas de dos nuevas escalas de medición fueron analizadas. El proceso de construcción de las escalas siguió los estándares para las pruebas educativas y psicológicas, así como manuales con las mejores prácticas para desarrollar y validar escalas.

\section{Participantes}

La muestra estuvo compuesta por grupos de investigación pertenecientes a IES colombianas. Estos grupos se encuentran registrados en la plataforma ScienTI, avalados por una institución y reconocidos por Colciencias en 2017. Para la obtención de la muestra se utilizó un muestreo no probabilístico, en tanto que, la selección de los participantes fue de tipo intencionado. En relación con el tamaño de muestra, se siguió la recomendación de Keith (2019), considerando un tamaño de muestra mínimo necesario de 200 participantes. La muestra empleada estuvo conformada por 334 grupos de investigación, donde la mayoría pertenece a la categoría C de Colciencias (39.222\%). Respecto a los años de antigüedad de los grupos, estas variaron desde los 3 hasta los 41 años $(M=$ $12.383, D E=6.185$ ). Por otro lado, el $31.595 \%$ tiene una naturaleza jurídica de corporaciones, el $57.362 \%$ pertenece al sector privado, el 50.613\% tiene un carácter académico, y el $38.922 \%$ centra su trabajo en el área de ciencias sociales. Respecto a las personas que respondieron las escalas, 263 (78.743\%) fueron líderes 0 directores del grupo de investigación, 57 investigadores (17.066\%), 7 líderes o directores de línea (2.096\%), 4 estudiantes de maestría (1.198\%), 2 estudiantes de doctorado $(0.599 \%)$ y un joven investigador $(0.299 \%)$.

\section{Instrumentos}

Los instrumentos de medición (Tabla 1) fueron presentados a los participantes de la siguiente manera: 1) consentimiento informado; 2) datos generales del grupo de investigación, donde se les solicitó el nombre del informante y el rol que cumple dentro del grupo de investigación; 3) escala de prácticas de gestión del conocimiento; y 4) escala de trabajo en equipo. Los instrumentos miden dos constructos: prácticas de gestión del conocimiento y trabajo en equipo. La primera escala está compuesta por dos factores: estrategia institucional (8 ítems) y creación del conocimiento (4 ítems). Por otro lado, la escala de trabajo en equipo está conformada por tres factores: comunidad de aprendizaje (10 ítems), colaboración y cooperación (9 ítems), y diversidad funcional (4 ítems). Las escalas estuvieron dirigidas a los líderes e investigadores de los grupos de investigación. La instrucción para los participantes, en cada escala, fue señalar su opinión en relación con las afirmaciones, evidenciando el número que mejor represente su respuesta del 1 (total desacuerdo) al 5 (total acuerdo). 
Tabla 1: Ítems de las escalas de medición.

\begin{tabular}{|c|c|}
\hline Código & Prácticas de Gestión Conocimiento (Ítem) \\
\hline PGCEI_1 & $\begin{array}{l}\text { La Universidad considera a los Grupos de Investigación como espacios de generación, gestión, y transferencia de } \\
\text { conocimiento. }\end{array}$ \\
\hline PGCEI_2 & $\begin{array}{l}\text { La Universidad orienta a su Grupo de Investigación a partir de una política de generación, gestión, y transferencia } \\
\text { de conocimiento e innovación. }\end{array}$ \\
\hline PGCEI_3 & La Universidad tiene un área de encargada de la gestión y transferencia de resultados de investigación. \\
\hline PGCEI_4 & $\begin{array}{l}\text { La Universidad tiene estrategias de apoyo para que su grupo de investigación realice acciones de transferencia de } \\
\text { resultados de investigación. }\end{array}$ \\
\hline PGCEI_5 & $\begin{array}{l}\text { La Universidad tiene convocatorias permanentes para el fomento de actividades de investigación e innovación en } \\
\text { los Grupos de Investigación. }\end{array}$ \\
\hline PGCEI_6 & La Universidad premia el desempeño sobresaliente en investigación de los Grupos de Investigación. \\
\hline PGCEI_7 & $\begin{array}{l}\text { La Universidad tiene políticas que fomentan escenarios de dialogo y colaboración entre su Grupo de Investigación, } \\
\text { la empresa, el Estado y las comunidades. }\end{array}$ \\
\hline PGCEI_8 & La Universidad asigna espacios en el portafolio de servicios institucional para los Grupos de Investigación. \\
\hline PGCCC_1 & $\begin{array}{l}\text { Su Grupo de Investigación implementa prácticas de gestión de conocimiento para el desarrollo de actividades de } \\
\text { investigación e innovación. }\end{array}$ \\
\hline PGCCC_2 & $\begin{array}{l}\text { Su Grupo de Investigación contempla estrategias para acelerar y mejorar el flujo de información con sus grupos de } \\
\text { interés. }\end{array}$ \\
\hline PGCCC_3 & Su Grupo de Investigación tiene asignación de tiempo institucional para la generación y desarrollo de ideas. \\
\hline PGCCC_4 & Su Grupo de Investigación aporta con sus resultados de investigación a la toma de decisiones institucional. \\
\hline Código & Trabajo en Equipo (Ítem) \\
\hline TECA_1 & Su Grupo de Investigación toma iniciativas para intercambiar ideas, prácticas, motivaciones y compromisos. \\
\hline TECA_2 & $\begin{array}{l}\text { Su Grupo de Investigación o el líder tiene comunicación directa con la alta gerencia de la universidad, para } \\
\text { movilizar y gestionar recursos. }\end{array}$ \\
\hline TECA_3 & En su grupo de investigación existe compromiso para llevar a cabo objetivos, metas y actividades. \\
\hline TECA_4 & Su grupo de investigación adapta y actualiza estrategias y metas para adaptarse a los cambios del entorno. \\
\hline TECA_5 & $\begin{array}{l}\text { Su grupo de investigación mantiene informado por el líder o el área de gestión de la Universidad, acerca de las } \\
\text { novedades que requieran cambios en la estrategia de trabajo. }\end{array}$ \\
\hline TECA_6 & Su Grupo de Investigación está siempre en la búsqueda de incrementar su capacidad de respuesta. \\
\hline TECA_7 & Su Grupo de Investigación tiene mecanismos de monitoreo de información en el entorno. \\
\hline TECA_8 & $\begin{array}{l}\text { Su Grupo de Investigación se caracteriza por articularse con otros grupos de investigación o equipos de trabajo de } \\
\text { la Universidad. }\end{array}$ \\
\hline TECA_9 & Su Grupo de Investigación contempla estrategias para prevenir conflictos o controversias. \\
\hline TECA_10 & Su Grupo de Investigación genera iniciativas para aprender de las equivocaciones y los éxitos. \\
\hline TECC_1 & $\begin{array}{l}\text { Su Grupo de Investigación tiene un directorio de conocimiento donde se muestra líneas, investigadores, proyectos } \\
\text { y resultados. }\end{array}$ \\
\hline TECC_2 & $\begin{array}{l}\text { Su Grupo de Investigación cuenta con una herramienta de gestión para la información que genera de las } \\
\text { actividades de investigación e innovación. }\end{array}$ \\
\hline TECC_3 & $\begin{array}{l}\text { Su Grupo de Investigación hace uso de herramientas tecnológicas para reducir el tiempo de gestión y desarrollo de } \\
\text { estrategias, metas o actividades. }\end{array}$ \\
\hline TECC_4 & Su Grupo de Investigación prefiere el trabajo colaborativo para sumar esfuerzos y disminuir riesgos. \\
\hline TECC_5 & Su Grupo de Investigación comparte recursos intelectuales y técnicos con sus grupos de interés. \\
\hline TECC_6 & $\begin{array}{l}\text { Su Grupo de Investigación ha transformado colectivamente resultados de investigación en productos, servicios, y } \\
\text { procesos con utilidad comprobada. }\end{array}$ \\
\hline TECC_7 & Usted conoce la especialidad y la experiencia de todos los miembros de su Grupo de Investigación. \\
\hline TECC_8 & Su Grupo de Investigación cuenta con metodologías para procesos de vigilancia tecnológica y comercial. \\
\hline TECC_9 & $\begin{array}{l}\text { Su grupo de investigación implementa estrategias para asegurar la transferencia de conocimiento a nuevos } \\
\text { investigadores o semilleros de investigación. }\end{array}$ \\
\hline TEDF_1 & $\begin{array}{l}\text { Existe confianza en los miembros de su Grupo de Investigación para desarrollar actividades, labores o proyectos } \\
\text { conjuntamente. }\end{array}$ \\
\hline TEDF_2 & $\begin{array}{l}\text { Los objetivos de su Grupo de Investigación están articulados con la identidad corporativa y el proyecto educativo } \\
\text { institucional de la Universidad. }\end{array}$ \\
\hline TEDF_3 & Los objetivos de su Grupo de Investigación son representativos y realizables para todos los integrantes. \\
\hline TEDF_4 & Para su Grupo de Investigación son importantes los resultados colectivos. \\
\hline
\end{tabular}


El proceso de construcción de ambas escalas inició con la definición conceptual y operacional de cada uno de los constructos de estudio. Seguidamente, se determinaron las dimensiones relevantes acorde a la perspectiva teórica desarrollada en la introducción y enfocadas en los grupos de investigación. Una vez especificada la estructura dimensional teórica de las dos escalas de medición, se procedió a la redacción de los ítems, con base en la experticia de los autores de los instrumentos y fundamentados en el marco teórico adoptado. Las versiones iniciales contaban con 18 ítems para la escala de prácticas de gestión del conocimiento y 24 ítems para la escala de trabajo en equipo. Posteriormente, se recolectó la evidencia de validez basada en el contenido de las escalas a partir de la opinión de ocho jueces expertos en las temáticas de gestión del conocimiento y trabajo en equipo dentro del contexto de investigación en educación superior. Sus recomendaciones permitieron modificar algunos ítems y eliminar aquellos menos representativos y relevantes de las variables a medir. Finalmente, se realizó un estudio piloto con las versiones preliminares de las escalas en 101 grupos de investigación, con la finalidad de mejorar la claridad y comprensión de los ítems por parte de la muestra de estudio. El estudio piloto permitió obtener las versiones finales de las escalas para la aplicación masiva.

\section{Procedimiento}

La recogida de información se realizó a través de un formulario electrónico, diseñado en la plataforma SurveyMonkey. Los instrumentos fueron aplicados a los líderes e investigadores de los grupos de investigación de IES colombianas a través de sus correos electrónicos institucionales o personales. Asimismo, los participantes del estudio recibieron un consentimiento informado donde se les invitó responder los instrumentos de manera voluntaria y confidencial, precisando el objetivo del estudio y su ámbito de aplicación. El tiempo de duración para la resolución de los instrumentos fueron entre 15 y 20 minutos aproximadamente. Los participantes no recibieron algún tipo de remuneración por su participación en el estudio.

\section{Análisis de datos}

El análisis estadístico se realizó con R, versión 4.0.2, a partir de los paquetes tidyverse, psych, lavaan y semTools. Primero, se realizó el análisis descriptivo de los ítems a través de la media, desviación estándar, asimetría y curtosis, considerando adecuados valores entre -1.5 y 1.5 para estos dos últimos. Asimismo, se obtuvieron los porcentajes de efectos suelo y techo, tomando como valores óptimos aquellos que se encontraran por debajo o iguales a 15\% (McHorney y Tarlov, 1995). Por otro lado, el nivel de discriminación de los ítems fue estimado a través de la correlación ítem-test, considerándose valores por encima de .30 como adecuados.

Segundo, se recurrieron a tres fuentes para obtener evidencias de validez; se recolectó evidencia de validez basada en el contenido de las pruebas, mediante el criterio de un grupo de jueces expertos. Asimismo, para aportar evidencia de validez basada en la estructura interna de las escalas, se empleó el análisis factorial confirmatorio (AFC). El AFC se realizó mediante el método de estimación de mínimos cuadrados ponderados con media y varianza ajustada (WLMSV), empleado por la robustez que presenta en el análisis de variables categóricas, tomando como insumo la matriz de correlaciones policóricas. Los índices de bondad de ajuste utilizados fueron: la razón entre $S S \chi^{2}$ y grados de libertad $\left(S S \chi^{2} / g l\right)$, donde se consideran apropiados valores menores a 3; el índice de ajuste comparativo (CFI), considerado adecuado alrededor de .95; el índice de TuckerLewis (TLI), tomando como adecuados los valores cercanos a .95; el error cuadrático medio de aproximación (RMSEA), donde valores menores a .08 fueron adecuados; el residuo estandarizado cuadrático medio (SRMR), donde fueron adecuados valores menores a .08; y el residuo ponderado cuadrático medio (WRMR), considerando un punto de corte de 1 como aceptable (DiStefano et al., 2018; Hu y Bentler, 1999; Keith, 2019).

Las evidencias de validez basada en la relación con otras variables se recolectaron a partir de la varianza media extraída (AVE), considerando como adecuados aquellos valores alrededor de .50, a esta fuente de información también se le conoce como evidencia convergente. Asimismo, la evidencia discriminante fue evaluada a partir de la ratio heterotrait-monotrait (HTMT), considerándose como valores apropiados por debajo de .95. Por último, se estimó la fiabilidad de consistencia interna mediante el coeficiente omega $(\omega)$, que considera el modelo obtenido a través del AFC. Se consideró como nivel satisfactorio un valor mayor a .70.

\section{RESULTADOS}

En esta sección se presentan los resultados de la recolección de evidencia de validez basada en el contenido de las escalas, el análisis descriptivo de los ítems, los análisis factoriales confirmatorios, la evidencia de validez convergente y el análisis de fiabilidad.

\section{Análisis descriptivos}

Respecto a los ítems de la escala de prácticas de gestión del conocimiento (Tabla 2), el ítem PGCEI_1 es el que mayor puntaje medio obtuvo, en tanto que, el ítem PGCCC_4 presentó el promedio más bajo. Por otro 
lado, el ítem PGCEI_3 mostró la mayor variabilidad en sus respuestas y el ítem PGCEI_1 la menor variabilidad. Los valores de asimetría y curtosis en todos los ítems se encontraron entre -1.5 y 1.5 , indicando que las distorsiones en las distribuciones de sus puntajes no son significativas. Además, no se reportó ningún efecto suelo en los ítems. No obstante, se observaron efectos techo en todos los ítems, es decir, más del 15\% de los participantes eligieron la puntuación más alta, siendo el ítem PGCEI_1 el que mayor efecto techo presentó (58\%). Finalmente, la correlación ítem-test en todos los ítems estuvo por encima de .30, yendo desde .489 (ítem PGCEI_5) hasta .742 (ítems PGCEI_2 y PGCEI_4), indicando una buena discriminación.

Tabla 2: Análisis de ítems de la escala de prácticas de gestión del conocimiento.

\begin{tabular}{|l|c|c|c|c|c|c|c|}
\hline \multicolumn{1}{|c|}{ İtem } & $M$ & $D E$ & Asimetría & Curtosis & Item-test & Suelo (\%) & Techo (\%) \\
\hline Estrategia Institucional & & & & & & & \\
\hline PGCEI_1 & 4.404 & 0.835 & -1.391 & 1.401 & .656 & 0 & 58 \\
\hline PGCEI_2 & 4.057 & 1.001 & -0.972 & 0.343 & .742 & 2 & 40 \\
\hline PGCEI_3 & 3.820 & 1.301 & -0.817 & -0.491 & .592 & 8 & 43 \\
\hline PGCEI_4 & 3.787 & 1.085 & -0.600 & -0.409 & .742 & 3 & 31 \\
\hline PGCEI_5 & 4.180 & 0.935 & -1.043 & 0.594 & .489 & 1 & 46 \\
\hline PGCEI_6 & 3.572 & 1.259 & -0.446 & -0.935 & .571 & 7 & 31 \\
\hline PGCEI_7 & 3.569 & 1.101 & -0.423 & -0.553 & .700 & 4 & 23 \\
\hline PGCEI_8 & 3.509 & 1.170 & -0.403 & -0.698 & .675 & 6 & 24 \\
\hline Creación de Conocimiento & & & & & & & \\
\hline PGCCC_1 & 3.656 & 1.001 & -0.439 & -0.381 & .629 & 2 & 21 \\
\hline PGCCC_2 & 3.551 & 0.981 & -0.322 & -0.418 & .678 & 2 & 17 \\
\hline PGCCC_3 & 3.494 & 1.296 & -0.482 & -0.885 & .561 & 10 & 28 \\
\hline PGCCC_4 & 3.446 & 1.171 & -0.447 & -0.655 & .626 & 7 & 20 \\
\hline
\end{tabular}

En relación con el análisis de ítems de la escala de trabajo en equipo (Tabla 3), el ítem TECC_7 es el que mayor puntaje promedio obtuvo. Por el contrario, el ítem TECC_8 presentó el promedio más bajo. En cuanto a la variabilidad, el ítem TECC_8 mostró la mayor dispersión en sus respuestas y el ítem TEDF_4 la menor variabilidad. Los coeficientes de asimetría y curtosis en todos los ítems se encontraron entre $-1 . \overline{5}$ y 1.5 , con excepción de los ítems TECC_7 y TEDF_4. Por otro lado, solo el ítem TECC_8 presentó efecto suelo (17\%). Sin embargo, se observaron efectos techo en todos los ítems, con excepción de los ítems TECA_7 (14\%), TECC_2 (15\%), TECC_3 (15\%) y TECC_8 (15\%); el ítem TECC_7 fue el que mayor efecto techo presentó $(71 \%)$. Por último, las correlaciones ítem-test fueron superiores a .30 en todos los ítems, indicativo de adecuados niveles de discriminación, encontrándose entre .342 (ítem TECC_7) y .669 (ítem TECA_6).

\section{Evidencia de validez basada en el contenido del test}

Se recurrió al criterio de ocho jueces expertos que brindaron sus recomendaciones para la mejora de las escalas. El grupo de expertos estuvo compuesto por un vicerrector de investigación de una IES; tres líderes de grupos de investigación categorizados en los niveles $\mathrm{B}$ y $\mathrm{C}$ de Colciencias; dos reconocidos académicos especialistas en las áreas de gestión del conocimiento, ciencia, tecnología e innovación; y dos gestores de investigación. Siete jueces pertenecían a tres universidades privadas colombianas y un juez a una universidad nacional colombiana. Se envió un correo electrónico adjuntando un documento que presentaba la contextualización del estudio, así como los factores e ítems para cada una de las escalas. La versión inicial de la escala de prácticas de gestión del conocimiento estuvo compuesta por 18 ítems, en tanto que, la escala de trabajo en equipo contenía 24 ítems. A partir de las observaciones brindadas por los jueces se realizaron modificaciones a algunos ítems y se eliminaron siete ítems por considerarse inadecuados (seis de la escala de prácticas de gestión del conocimiento y uno de la escala de trabajo en equipo).

\section{Evidencia de validez basada en la estructura interna del test}

EI AFC realizado para las escalas de prácticas de gestión del conocimiento y trabajo en equipo, indicó un buen ajuste para los modelos propuestos a nivel teórico. En específico, para la escala de prácticas de gestión del conocimiento se puso a prueba cuatro modelos, siendo el modelo oblicuo D (factores correlacionados) el que mejores índices de ajuste presentó (Tabla 4). Asimismo, las cargas factoriales de sus ítems fueron significativas y superiores a .50 , con un valor mínimo de .584 y un valor máximo de .887 (Fig. 1). Finalmente, la correlación entre sus dimensiones fue grande (.720). Respecto a la escala de trabajo en equipo, se probaron seis modelos, donde el modelo oblicuo $\mathrm{F}$ fue el que mejores índices de ajuste mostró (Tabla 4). En este 
modelo, se eliminó el ítem TECA_2 de la dimensión comunidad de aprendizaje y los ítems TECC_2 y TECC_7 de la dimensión colaboración y cooperación; todos ellos por tener altos índices de modificación. Por otro lado, las cargas de los ítems se encontraron entre .582 y .875, siendo todas significativas (Fig. 2). Además, las correlaciones entre sus dimensiones, indicaron una fuerte relación entre ellas, debido a que se encontraron entre .752 y .946 (Fig. 2). Ambos resultados del AFC aportan evidencia de validez basada en la estructura interna de las escalas de prácticas de gestión del conocimiento y trabajo en equipo, dado que existe una congruencia entre el modelo teórico propuesto y los datos empíricos recolectados.

Tabla 3: Análisis de ítems de la escala de trabajo en equipo.

\begin{tabular}{|l|c|c|c|c|c|c|c|}
\hline \multicolumn{1}{|c|}{ Ítem } & $M$ & $D E$ & Asimetría & Curtosis & Ítem-test & Suelo (\%) & Techo (\%) \\
\hline Comunidad de Aprendizaje & & & & & & & \\
\hline TECA_1 & 3.979 & 0.888 & -0.548 & -0.234 & .626 & 1 & 32 \\
\hline TECA_2 & 3.557 & 1.223 & -0.524 & -0.696 & .445 & 7 & 27 \\
\hline TECA_3 & 4.129 & 0.883 & -1.036 & 1.032 & .649 & 1 & 38 \\
\hline TECA_4 & 3.928 & 0.930 & -0.772 & 0.329 & .654 & 1 & 29 \\
\hline TECA_5 & 3.898 & 1.055 & -0.792 & -0.049 & .644 & 3 & 34 \\
\hline TECA_6 & 4.105 & 0.879 & -0.680 & -0.249 & .669 & 0 & 39 \\
\hline TECA_7 & 3.317 & 1.088 & -0.247 & -0.701 & .660 & 5 & 14 \\
\hline TECA_8 & 3.856 & 1.033 & -0.723 & -0.038 & .550 & 3 & 31 \\
\hline TECA_9 & 3.473 & 1.106 & -0.384 & -0.609 & .579 & 5 & 19 \\
\hline TECA_10 & 3.751 & 0.978 & -0.431 & -0.556 & .654 & 1 & 25 \\
\hline Colaboración y Cooperación & & & & & & & \\
\hline TECC_1 & 3.740 & 1.147 & -0.528 & -0.631 & .558 & 4 & 34 \\
\hline TECC_2 & 3.141 & 1.204 & -0.106 & -0.897 & .563 & 10 & 15 \\
\hline TECC_3 & 3.302 & 1.137 & -0.291 & -0.702 & .643 & 7 & 15 \\
\hline TECC_4 & 4.249 & 0.864 & -1.084 & 0.937 & .509 & 1 & 47 \\
\hline TECC_5 & 4.012 & 0.923 & -0.776 & 0.235 & .599 & 1 & 34 \\
\hline TECC_6 & 3.701 & 1.120 & -0.672 & -0.244 & .575 & 5 & 27 \\
\hline TECC_7 & 4.626 & 0.672 & -1.945 & 3.673 & .342 & 0 & 71 \\
\hline TECC_8 & 2.868 & 1.226 & 0.017 & -0.957 & .500 & 17 & 10 \\
\hline TECC_9 & 3.889 & 0.988 & -0.710 & 0.056 & .546 & 2 & 31 \\
\hline Diversidad Funcional & & & & & & & \\
\hline TEDF_1 & 4.362 & 0.804 & -1.226 & 1.361 & .551 & 1 & 53 \\
\hline TEDF_2 & 4.482 & 0.726 & -1.301 & 1.129 & .498 & 0 & 60 \\
\hline TEDF_3 & 4.410 & 0.703 & -0.919 & 0.135 & .641 & 0 & 53 \\
\hline TEDF_4 & 4.599 & 0.663 & -1.692 & 2.996 & .606 & 0 & 69 \\
\hline
\end{tabular}

\section{Evidencia de validez basada en la relación con otras variables}

Para esta fuente de evidencia de validez se recurrió a la evidencia convergente. El método estadístico utilizado fue el AVE, que indica la proporción de varianza promedio que explica una variable a partir de las cargas factoriales de los ítems que la componen. En la Tabla 5 se puede observar que las dos dimensiones de la escala de prácticas de gestión del conocimiento tienen un AVE mayor a .50, es decir, más del $50 \%$ de la varianza explicada por las dimensiones no se debe al error de medición. Por otro lado, las dimensiones de la escala de trabajo en equipo también se encuentran por encima de .50, con excepción de colaboración y cooperación (AVE $=.461)$, aunque su valor está ligeramente por debajo del criterio asumido. Además, la evidencia discriminante se valoró mediante la ratio HTMT que implica el promedio de todas las correlaciones entre los ítems de las distintas variables con relación a la media geométrica del promedio de las correlaciones entre los ítems que miden una misma variable. En todos los casos, la ratio HTMT estuvo por debajo de .95, encontrándose entre .531 (estrategia institucional y diversidad funcional) y .939 (comunidad de aprendizaje y colaboración y cooperación). De estos resultados, se concluye que las interpretaciones a partir de las puntuaciones en las escalas cuentan con evidencias de validez basada en la relación con otras variables. 
Tabla 4: Resultados del AFC de las escalas de prácticas de gestión del conocimiento y trabajo en equipo.

\begin{tabular}{|c|c|c|c|c|c|c|c|c|}
\hline Modelo & $S S \chi^{2}$ & $g l$ & $S S \chi^{2} / g I$ & RMSEA [IC 90\%] & CFI & TLI & SRMR & WRMR \\
\hline \multicolumn{9}{|c|}{ Prácticas de gestión del conocimiento } \\
\hline A: Jerárquico & 136.589 & 52 & 2.627 & $.070[.056 ; .084]$ & .983 & .979 & .050 & 1.095 \\
\hline B: Ortogonal & 1495.235 & 54 & 27.690 & $.283[.271 ; .296]$ & .715 & .652 & .243 & 4.692 \\
\hline C: Oblicuo-c & 208.656 & 53 & 3.937 & $.094[.081 ; .107]$ & .969 & .962 & .050 & 1.095 \\
\hline D: Oblicuo-d & 148.628 & 52 & 2.858 & $.075[.061 ; .089]$ & .981 & .976 & .040 & 0.881 \\
\hline \multicolumn{9}{|c|}{ Trabajo en equipo } \\
\hline A: Jerárquico & 919.920 & 227 & 4.053 & $.096[.089 ; .102]$ & .910 & .899 & .075 & 1.489 \\
\hline B: Ortogonal & 5747.761 & 230 & 24.990 & $.268[.262 ; .274]$ & .280 & .208 & .324 & 6.507 \\
\hline C: Oblicuo-c & 919.920 & 227 & 4.053 & $.096[.089 ; .102]$ & .910 & .899 & .075 & 1.489 \\
\hline D: Oblicuo-d & 738.466 & 205 & 3.602 & $.088[.082 ; .095]$ & .928 & .919 & .067 & 1.357 \\
\hline E: Oblicuo-e & 600.872 & 185 & 3.248 & $.082[.075 ; .090]$ & .942 & .934 & .062 & 1.237 \\
\hline F: Oblicuo-f & 505.794 & 166 & 3.047 & $.078[.071 ; .086]$ & .951 & .943 & .057 & 1.153 \\
\hline
\end{tabular}

Tabla 5: Análisis descriptivo medio y fiabilidad de las escalas de prácticas de gestión del conocimiento y trabajo en equipo.

\begin{tabular}{|l|c|c|c|c|c|c|}
\hline \multicolumn{1}{|c|}{ Variable } & $n$ & $M$ & $D E$ & Correlación inter-ítem & AVE & $\omega$ \\
\hline Prácticas de gestión del conocimiento & & & & & & \\
\hline Estrategia institucional & 8 & 3.862 & .803 & .482 & .581 & .903 \\
\hline Creación de conocimiento & 4 & 3.537 & .886 & .517 & .543 & .763 \\
\hline Trabajo en equipo & & & & & & \\
\hline Comunidad de aprendizaje & 9 & 3.826 & .708 & .461 & .533 & .890 \\
\hline Colaboración y cooperación & 7 & 3.680 & .727 & .384 & .461 & .823 \\
\hline Diversidad funcional & 4 & 4.463 & .558 & .460 & .604 & .797 \\
\hline
\end{tabular}

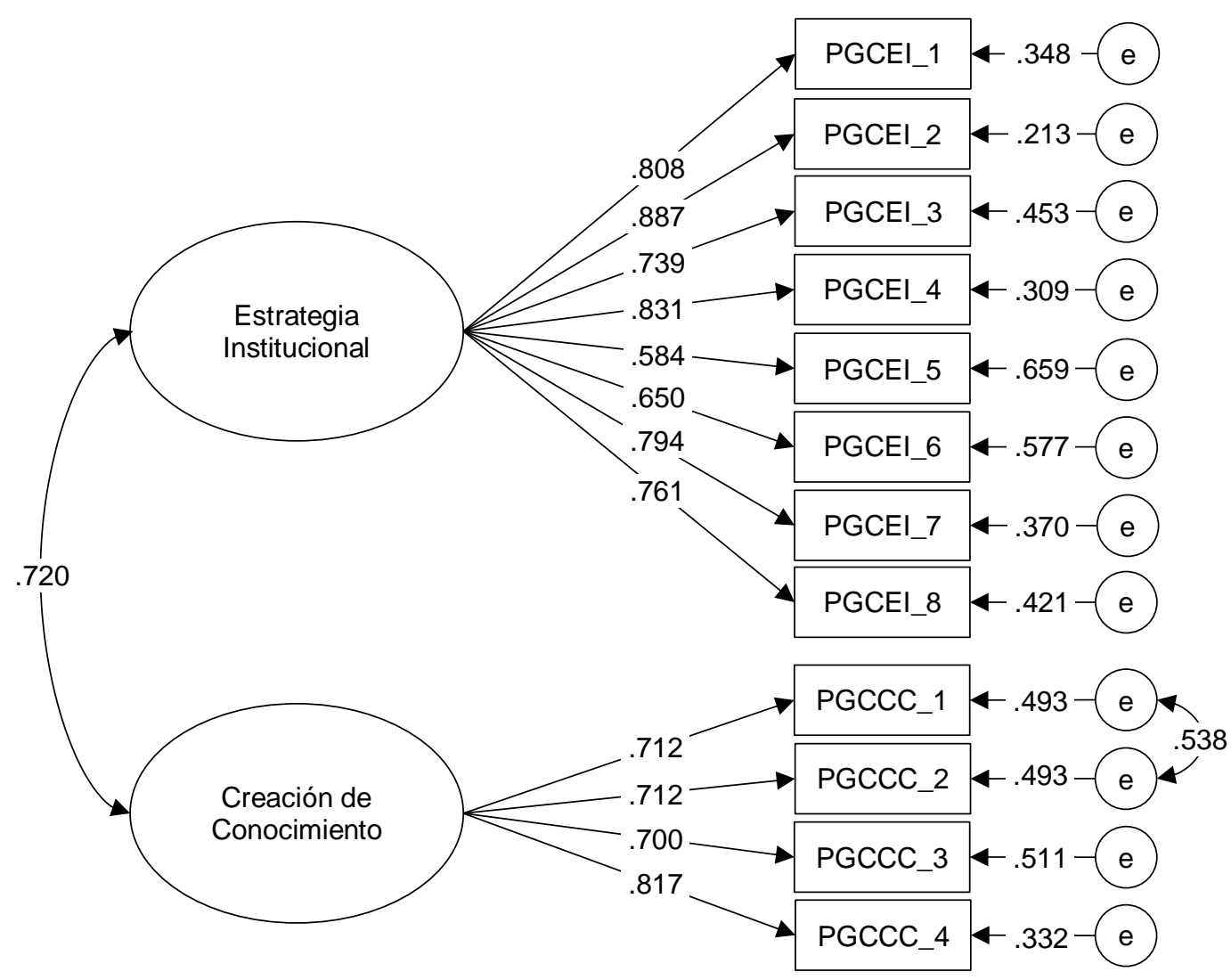

Fig. 1: Coeficientes estandarizados de la escala de prácticas de gestión del conocimiento. 


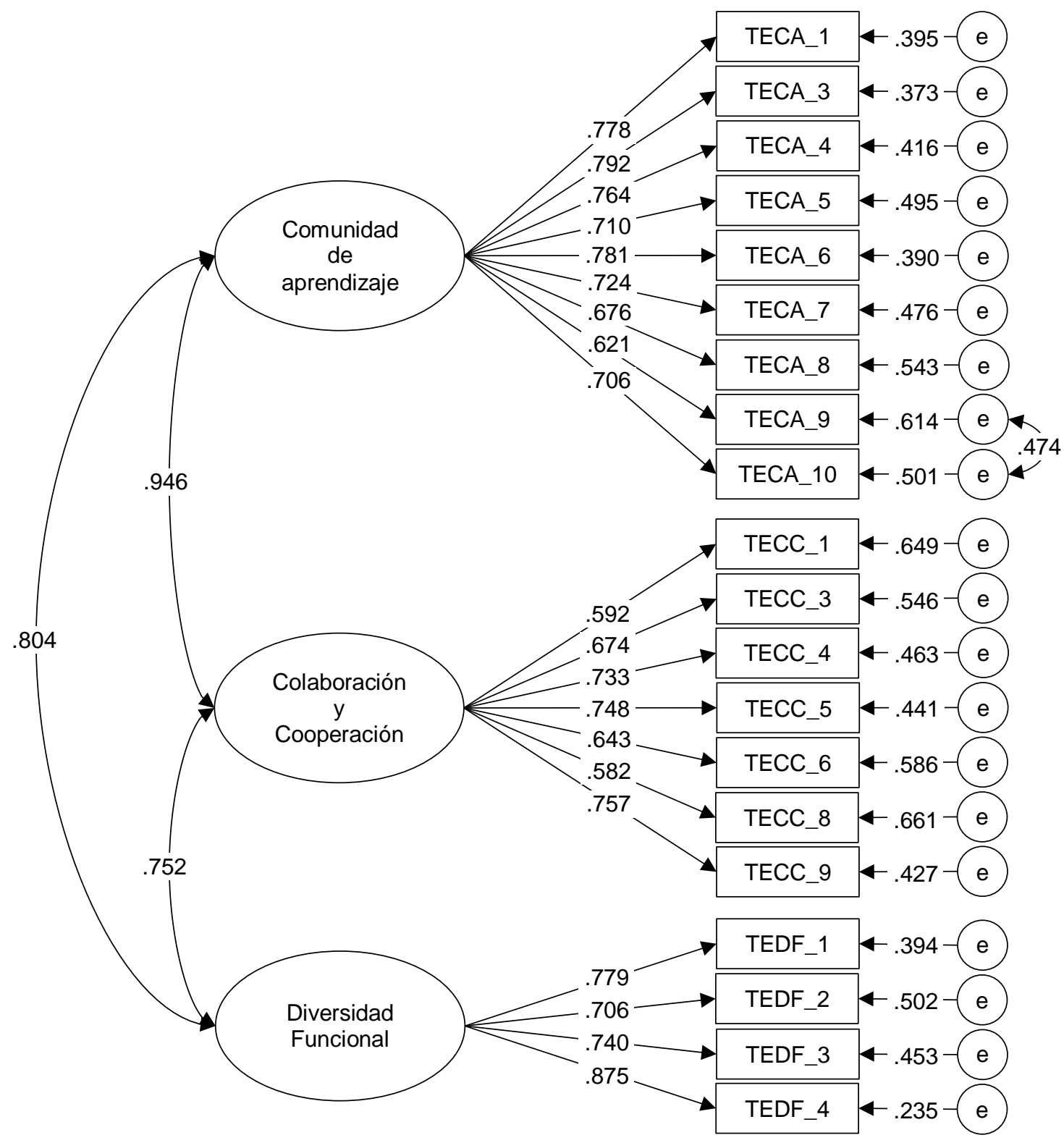

Fig. 2: Coeficientes estandarizados de la escala de trabajo en equipo.

\section{DISCUSIÓN}

Las escalas desarrolladas son instrumentos de autoreporte que miden dos constructos, las prácticas de gestión del conocimiento y el trabajo en equipo, variables claves en el funcionamiento y productividad de los grupos de investigación. Ambas escalas fueron revisadas por un grupo de jueces expertos que brindaron evidencia de validez basada en el contenido en función a la relevancia y representatividad de los ítems. De acuerdo con los resultados del modelo estructural, la escala de prácticas de gestión del conocimiento está formada por dos dimensiones: estrategia institucional y creación del conocimiento; en tanto que, la escala de trabajo en equipo está compuesta por tres factores: comunidad de aprendizaje, colaboración y cooperación, y diversidad funcional. Adicionalmente, ambas pruebas cuentan con evidencia de validez basada en la relación con otras variables, tanto evidencia convergente como discriminante. En relación con la consistencia interna de las puntuaciones de las escalas, muestra una adecuada fiabilidad a través del coeficiente omega, tanto para las dimensiones de la escala de prácticas de gestión del conocimiento como para las dimensiones de la escala de trabajo en equipo.

En la actualidad no existen instrumentos validados que midan las prácticas de gestión del conocimiento en grupos de investigación, por lo que, la escala desarrollada permite evaluar este constructo en esta muestra relevante en la sociedad del conocimiento. La escala que guarda mayor relación con el presente estudio fue la construida por Guevara (2011), que mide las prácticas de gestión del conocimiento en grupos de investigación desde una perspectiva unidimensional considerando cuatro prácticas: de socialización, exteriorización, combinación e interiorización. Sin embargo, el instrumento no presenta información sobre sus propiedades psicométricas, por lo que no es pertinente su utilización sin conocer su funcionamiento, peor aún 
en situaciones donde las evaluaciones en grupos de investigación sirven, por ejemplo, para la toma de decisiones en políticas institucionales como pueden ser los financiamientos de proyectos de investigación, implementación en recursos tecnológicos o inversión en infraestructura. Asimismo, la escala desarrollada por Paez-Logreira et al. (2016) que mide las percepciones de la gestión del conocimiento en grupos de investigación presenta las mismas carencias que el instrumento de Guevara (2011). Por el contrario, la escala aquí presentada cuenta con evidencias de validez a partir de tres fuentes, así como evidencias de fiabilidad mediante la consistencia interna de las respuestas en los ítems propuestos.

Estos niveles validez y fiabilidad obtenidos en la escala de prácticas de gestión del conocimiento fueron similares a los reportados por Acosta-Prado et al. (2020) que midieron las condiciones de la gestión del conocimiento, vistas como factores que influyen o ayudan en el manejo del conocimiento, en un grupo de empresas con base tecnológica. Por otro lado, el estudio de Lee y Wong (2015) midió la estrategia y creación-generación de conocimiento mediante tres y cuatro ítems, respectivamente, en un grupo de pequeñas y medianas empresas; obteniendo una fiabilidad aceptable para ambos factores y un AVE de .838 para estrategia y de .603 para creación-generación de conocimiento. Finalmente, Singh y Gupta (2014) evaluaron la creación de conocimiento por medio de nueve ítems en un grupo de profesionales del campo de la tecnología e información, donde su nivel de fiabilidad fue adecuado; no obstante, tres cargas factoriales fueron menores a .40.

Respecto a la escala de trabajo en equipo, no se encontraron instrumentos que midan esta variable en grupos de investigación. Por lo que, la escala desarrollada ayudará en la medición de este constructo en el contexto de investigación dentro de las IES. Con relación a otros instrumentos que miden alguno de los factores del trabajo en equipo, se encontró que, Ramayah et al. (2014) evalúan las comunidades de práctica a través de siete ítems, que presentan una correlación ítem-test por encima de .40 y un adecuado nivel de fiabilidad. Además, Nikolova et al. (2014) midieron el aprendizaje con colegas, encontrando un buen nivel de fiabilidad. Los estudios de Mendo-Lázaro et al. (2017), Aguado et al. (2014) y Gastélum et al. (2012) reportaron escalas que miden distintos aspectos del trabajo en equipo, actitudes, competencias y autoeficacia, respectivamente, en muestras distintas a la trabajada en este estudio. No obstante, los niveles de validez y fiabilidad reportados presentan coincidencias a lo hallado en la escala construida. Estos resultados brindan soporte a lo encontrado en el presente estudio, tanto en el análisis de ítems como en las evidencias de fiabilidad y validez.

En relación a las limitaciones del estudio, el principal está relacionado al tamaño de muestra, que limita el uso de algunos análisis estadísticos que podrían brindan evidencias de validez adicionales, por ejemplo, la invarianza factorial o el funcionamiento diferencial de los ítems. No obstante, la conceptualización actual de validez entiende a esta como un proceso continuo de acumulación de evidencias que justifiquen las interpretaciones y usos de un instrumento, por lo que, la metodología utilizada en este estudio permite obtener las primeras evidencias para estas escalas de medición, permitiendo su aplicación en grupos de investigación. Finalmente, el desarrollo de estas dos escalas pone en relieve la importancia que ha ido adquiriendo la investigación en todos los ámbitos de la vida, siendo necesarias herramientas para comprender el funcionamiento de los grupos de investigación quienes son los principales actores en la generación de conocimiento en las sociedades. Estos instrumentos de medición pueden ser utilizados por aquellas entidades encargadas de mejorar la calidad investigativa de las IES, así como los gestores universitarios para determinar el nivel de implementación de políticas a favor de la producción científica y detectar posibles desviaciones que sirvan para mejorar el funcionamiento en sus respectivos ámbitos de trabajo, con el objetivo final de impulsar el desarrollo científico y tecnológico de un país.

\section{CONCLUSIONES}

Teniendo en cuenta los resultados obtenidos se deduce que, las escalas de medida creadas para cada constructo (prácticas de gestión del conocimiento y trabajo en equipo) cumplen con las propiedades psicométricas relacionadas a la fiabilidad y validez exigidas en los instrumentos de medición en ciencias sociales. Por tanto, las escalas propuestas resultan idóneas para medir los constructos analizados en IES colombianas.

\section{REFERENCIAS}

Acosta-Prado, J.C., Romero, A.K. y Tafur-Mendoza, A.A., Conditions of Knowledge Management, Innovation Capability and Firm Performance in Colombian NTBFs: A Measurement Scale, https://doi.org/10.1108/VJIKMS-09-2019-0142, VINE J. Inf. Knowl. Manag. Syst (2020)

Aguado, D., Rico, R., Sánchez-Manzanares, M. y Salas, E., Teamwork Competency Test (TWCT): A Step Forward on Measuring Teamwork Competencies, https://doi.org/10.1037/a0036098, Group. Dyn., 18(2), 101-121 (2014)

Argueta, G.V.M. y Jiménez, C.P., Gestión del Conocimiento en Investigadores de la Universidad de Guadalajara (México), https://doi.org/10.24320/redie.2017.19.3.1151, Rev. Electrónica Investig. Educ., 19(3), 1-9 (2017)

Ato, M., López, J.J. y Benavente, A., A Classification System for Research Designs in Psychology, https://doi.org/10.6018/analesps.29.3.178511, An. Psicol., 29(3), 1038-1059 (2013) 
Casimir, G., Lee, K. y Loon, M., Knowledge Sharing: Influences of Trust, Commitment and Cost, https://doi.org/10.1108/13673271211262781, J. Knowl. Manag., 16(5), 740-753 (2012)

Colciencias, Modelo de Medición de Grupos de Investigación, Desarrollo Tecnológico o de Innovación y de Reconocimiento de Investigadores del Sistema Nacional de Ciencia, Tecnología e Innovación, Colciencias, Colombia (2018)

Conchado, A., Carot, J.M. y Bas, M.C., Competencies for Knowledge Management: Development and Validation of a Scale, https://doi.org/10.1108/JKM-10-2014-0447, J. Knowl. Manag., 19(4), 836-855 (2015)

DiStefano, C., Liu, J., Jiang, N. y Shi, D., Examination of the Weighted Root Mean Square Residual: Evidence for Trustworthiness? https://doi.org/10.1080/10705511.2017.1390394, Struct. Equ. Model. A Multidiscip. J., 25(3), 453-466 (2018)

García-Alisina, M. y Gómez-Vargas, M., Prácticas de Gestión del Conocimiento en los Grupos de Investigación: Estudio de un Caso, Rev. Interam. Bibl., 38(1), 13-25 (2015)

Gastélum, G., Guedea, J.C., Viciana, J. y Peinado, J.E., Composición Factorial de una Escala de Autoeficacia en el Ámbito del Trabajo en Equipo y Liderazgo en Universitarios de Ciencias de la Salud, https://doi.org/10.4067/S071850062012000400006, Form. Univ., 5(4), 49-60 (2012)

Gaviria, M.M., Mejía, A.M. y Henao, D.L., Gestión del Conocimiento en los Grupos de Investigación de Excelencia de la Universidad de Antioquia, Rev. Interam. Bibl., 30(2), 137-163 (2007)

Gómez, A. y Acosta, H., Acerca del Trabajo en Grupos o Equipos, Acimed, 11(6) (2003)

Guevara, P.C., La Gestión del Conocimiento en Grupos de Investigación de la Universidad Nacional de Colombia. Caso: Instituto de Biotecnología, Tesis de Magister, Fac. de Cien. Econ., Universidad Nacional de Colombia, Colombia (2011)

Hu, L. y Bentler, P.M., Cutoff Criteria for Fit Indexes in Covariance Structure Analysis: Conventional Criteria versus New Alternatives, https://doi.org/10.1080/10705519909540118, Struct. Equ. Model., 6(1), 1-55 (1999)

Hussinki, H., Kianto, A., Vanhala, M. y Ritala, P., Assessing the Universality of Knowledge Management Practices, https://doi.org/10.1108/JKM-09-2016-0394, J. Knowl. Manag., 21(6), 1596-1621 (2017)

Keith, T.Z., Multiple Regression and Beyond: An Introduction to Multiple Regression and Structural Equation Modeling (3rd ed.), Routledge, New York (2019)

Lee, C.S. y Wong, K.Y., Development and Validation of Knowledge Management Performance Measurement Constructs for Small and Medium Enterprises, https://doi.org/10.1108/JKM-10-2014-0398, J. Knowl. Manag., 19(4), 711-734 (2015)

Lin, K.Y., Hong, H.-Y. y Chai, C.S., Development and Validation of the Knowledge-Building Environment Scale, https://doi.org/10.1016/j.lindif.2013.10.018, Learn. Individ. Differ., 30, 124-132 (2014)

McHorney, C.A., y Tarlov, A.R., Individual-Patient Monitoring in Clinical Practice: Are Available Health Status Surveys Adequate? https://doi.org/10.1007/BF01593882, Qual. Life Res., (4), 293-307 (1995)

Mendo-Lázaro, S., Polo-del-Río, M.I. y otros 3 autores, Construction and Validation of a Measurement Instrument for Attitudes towards Teamwork, https://doi.org/10.3389/fpsyg.2017.01009, Front. Psychol., 8, 1009 (2017)

Nikolova, I., Van Ruysseveldt, J., De Witte, H. y Syroit, J., Work-Based Learning: Development and Validation of a Scale Measuring the Learning Potential of the Workplace (LPW), https://doi.org/10.1016/j.jvb.2013.09.004, J. Vocat. Behav., 84(1), 1-10 (2014)

Paez-Logreira, H., Zamora-Musa, R. y Velez-Zapata, J., Relation Analysis of Knowledge Management, Research, and Innovation in University Research Groups, https://doi.org/10.4067/S0718-27242016000400002, Int. J. Technol. Manag., 11(4), 5-11 (2016)

Ramayah, T., Yeap, J.A.L. y lgnatius, J., Assessing Knowledge Sharing among Academics: A Validation of the Knowledge Sharing Behavior Scale (KSBS), https://doi.org/10.1177/0193841X14539685, Eval. Rev., 38(2), 160-187 (2014)

Ramírez, J.A., Alejo, M.G., Jiménez, R. y Marmolejo, S., Percepción de los Estudiantes de Ciencias Químicas sobre sus Equipos de Trabajo, https://doi.org/10.4067/S0718-50062013000300002, Form. Univ., 6(3), 3-12 (2013)

Rey, J., Martín, M.J. y Sebastián, J., Estructura y Dinámica de los Grupos de Investigación, Arbor, (732), $743-757$ (2008)

Rodríguez, A., Araujo, A. y Urrutia, J., La Gestión del Conocimiento Científico-Técnico en la Universidad: Un Caso y un Proyecto, Cuad. Gest., 1(1), 13-30 (2001)

Romo, A.E., Villalobos, M.A. y Guadalupe, L.E., Gestión del Conocimiento: Estrategia para la Formación de Investigadores, Sinéctica, (38) (2012)

Singh, R.M. y Gupta, M., Knowledge Management in Teams: Empirical Integration and Development of a Scale, https://doi.org/10.1108/JKM-11-2013-0450, J. Knowl. Manag., 18(4), 777-794 (2014)

Torres, M., Cruz, I. y Hernández, J., Gestión del Conocimiento: Experiencias de Instituciones Académicas y Hospitalarias, https://doi.org/10.12804/revsalud12.2.2014.03, Rev. Ciencias la Salud, 12(2), 169-181 (2014) 